\title{
A Simple and Improved HPLC-PDA Method for Simultaneous Estimation of Fexofenadine and Pseudoephedrine in Extended Release Tablets by Response Surface Methodology
}

\author{
Ruhul Kayesh, ${ }^{1}$ A. S. M. Moniruzzaman Sarker, ${ }^{1}$ \\ Md. Zakir Sultan, ${ }^{2}$ and Md. Sarowar Jahan ${ }^{3}$ \\ ${ }^{1}$ Department of Pharmaceutical Chemistry, University of Dhaka, Dhaka, Bangladesh \\ ${ }^{2}$ Centre for Advanced Research in Sciences, University of Dhaka, Dhaka, Bangladesh \\ ${ }^{3}$ Department of Biochemistry and Molecular Biology, University of Dhaka, Dhaka, Bangladesh \\ Correspondence should be addressed to Md. Sarowar Jahan; sjsojib@gmail.com
}

Received 14 July 2016; Revised 8 November 2016; Accepted 27 November 2016; Published 4 January 2017

Academic Editor: Pranav S. Shrivastav

Copyright (C) 2017 Ruhul Kayesh et al. This is an open access article distributed under the Creative Commons Attribution License, which permits unrestricted use, distribution, and reproduction in any medium, provided the original work is properly cited.

\begin{abstract}
A simple RP-HPLC method has been developed for simultaneous estimation of fexofenadine and pseudoephedrine in their extended release tablet. The method was developed based on statistical design of experiments (DoE) and Response Surface Methodology. Separation was achieved on double end-capped $\mathrm{C}_{18}$ column $(250 \mathrm{~mm} \times 4 \mathrm{~mm}, 5 \mu \mathrm{m})$. In this experiment, two components of mobile phase, namely, acetonitrile $(\% \mathrm{v} / \mathrm{v})$ and methanol $(\% \mathrm{v} / \mathrm{v})$, were the factors whereas retention and resolution of the chromatographic peaks were the responses. The effects of different composition of factors on the corresponding responses were investigated. The optimum chromatographic condition for the current case was found as an isocratic mobile phase consisting of $20 \mathrm{mM}$ phosphate buffer ( $\mathrm{pH} 6.8$ ) and acetonitrile and methanol in a ratio of $50: 36: 14(\% \mathrm{v} / \mathrm{v})$ at a flow rate of $1 \mathrm{~mL} / \mathrm{min}$ for 7 minutes. The retention of pseudoephedrine and fexofenadine was found to be $2.6 \mathrm{~min}$ and $4.7 \mathrm{~min}$, respectively. The method was validated according to the ICH and FDA guidelines and various validation parameters were determined. Also, forced degradation studies in acid, base, oxidation, and reduction media and in thermal condition were performed to establish specificity and stabilityindicating property of this method. Practical applicability of this method was checked in extended release tablets available in Bangladeshi market.
\end{abstract}

\section{Introduction}

Fexofenadine hydrochloride (FEX) is a nonsedating antihistamine which works by blocking the effects of histamine, a substance in the body that is primarily responsible for allergy symptoms [1,2]. Pseudoephedrine hydrochloride (PSU) is in the class of medications called decongestants. It is a directand indirect-acting sympathomimetic drug which works by drying up the nasal passages [1]. The combination of FEX and PSU is used in adults and children (12 years of age and older) to relieve the allergy symptoms of seasonal allergic rhinitis ("hay fever"), including runny nose, sneezing, congestion, or itching of the nose, throat, or roof of the mouth; and also for red, itchy, or watery eyes [3]. The combination of these two drugs comes as an extended release tablet formulation with either 12-hour or 24-hour release profile [4].
Appropriate and easy to use analytical method is of prime importance in pharmaceutical company to ensure the quality of the tablet dosage form with respect to assay, content uniformity, and dissolution during formulation development and in quality control of commercial batches. Few HPLC methods have been described elsewhere for simultaneous determination of these two drugs in their extended release tablet forms by HPLC, but all those methods lack some necessary information and basic elements of validation process as well as some essential features of a good HPLC method.

For example, Induri et al. described confusing $\mathrm{pH}$ of mobile phase buffer. In abstract $\mathrm{pH}$ was mentioned 2.8 but in the text it was 6.8 [5]. Whatever it is, it was necessary to mention the molar concentration of buffer salt as at that high organic percentage ( $60 \%$ methanol) salt precipitation is 
likely to happen (e.g., salt conc. $\geq 50 \mathrm{mM}$ ). In addition to this, the resolution seems not so good. Also a forced degradation study has been absent to establish its specificity and stabilityindicating property.

Karakuş et al. have chosen a $\mathrm{pH}$ of 4.5 of their mobile phase [6]. As a rule of thumb $\mathrm{pH}$ of mobile phase should be at least 2 units above or below the pKa value of ionizable (acidic/basic) drug to ensure that only one form of analyte exists in solution $[7,8]$. From this point of view, $\mathrm{pH}$ selection in this method is a weak point as FEX possesses a pKa value of 4.28. Secondly, in this method PSU eluted at about 1 minute which is another weak point as at lower UV range (218 in this case) the early elution often encounters interference from system peaks.

Sharma and Shah described a method wherein they used dioctyl sulfosuccinate as mobile phase buffer [9]. But to the best of our knowledge, no reference has been found describing the use of dioctyl sulfosuccinate as mobile phase buffer. In fact, dioctyl sulfosuccinate is a therapeutic agent used as laxative $[10,11]$. As an excipient, it is used as an emulsifier, wetting agent, and also in tablet manufacturing as lubricant $[11,12]$.

In the USP 2015 an ion-pair RP-HPLC method has been described for both assay and dissolution process [13]. In assay method the use of $16.3 \mathrm{~g} / \mathrm{L}$ of $\mathrm{n}$-octane sulphonic acid sodium salt $(75 \mathrm{mM})$ seems to be astronomically high for the HPLC columns. This high concentration of ion-pairing agent is detrimental to reversed phase column because ionpairing agents are not easily or completely washed out. There are other complications too. For example, it uses two types of columns (L6 and L11) connected in series, temperature is to be maintained at $35^{\circ} \mathrm{C}$, and $\mathrm{pH}$ of assay mobile phase is 4.6 which is close to pKa value of FEX.

In our current research work we put effort to develop a simple and robust RP-HPLC method which would be common for assay and dissolution rate determination without any ion-pairing agent or other complications. In the development process we successfully applied "Response Surface Methodology," the modern approach of HPLC method optimization. Also, the developed method was validated according to the ICH, USP, and FDA guideline.

\section{Materials and Method}

2.1. Materials. The working standards were kind gift from ACI Limited, Bangladesh. HPLC grade acetonitrile and methanol were obtained from Active Fine Chemicals Ltd., Bangladesh.

2.2. Standard and Sample Solution Preparation. Working standard of $60 \mathrm{mg}$ of PSU and $30 \mathrm{mg}$ of FEX were taken in a $100 \mathrm{~mL}$ volumetric flask and $5 \mathrm{~mL}$. Methanol was added to dissolve the mass; then it was filled to the mark with mobile phase. This was the stock standard solution. Suitable serial dilutions were made to get the nominal concentration $(30 \mu \mathrm{g} / \mathrm{mL}$ PSU and $15 \mu \mathrm{g} / \mathrm{mL}$ FEX) and other 5 different concentrations from $50 \%$ to $200 \%$ of the nominal.

For assay preparation, 20 tablets were grinded and tablet powder equivalent to $60 \mathrm{mg}$ of PSU and $30 \mathrm{mg}$ of FEX were
TABLE 1: Types of degradation reactions and conditions.

\begin{tabular}{ll}
\hline Degradation reaction & Typical conditions \\
\hline Thermal degradation & Heated at $105^{\circ} \mathrm{C}$ for three hours \\
Acid hydrolysis & Treated with $1 \mathrm{~N} \mathrm{HCl}$ up to 24 hours \\
Base hydrolysis & Treated with $1 \mathrm{~N} \mathrm{NaOH}$ up to 24 hours \\
Oxidation & Treated with $10 \% \mathrm{H}_{2} \mathrm{O}_{2}$ solution up to 24 \\
hours \\
Reduction & Treated with $10 \% \mathrm{Na}$ bisulfite solution up \\
& to 24 hours
\end{tabular}

taken in a $100 \mathrm{~mL}$ volumetric flask and suitably diluted with mobile phase. Complete dissolution of drugs in the diluting solvent was achieved by proper sonication.

For dissolution sample, dissolution media containing the drugs were filtered through $0.45 \mu \mathrm{m}$ filter paper and the filtrate was diluted with mobile phase.

2.3. Validation. The developed method was validated according to the ICH, USP, and FDA guidelines with respect to accuracy, precision, specificity, system suitability, LOD, LOQ, and robustness [14-16].

2.4. Forced Degradation Study. Forced degradation studies are undertaken to degrade the sample (e.g., drug product or drug substance) deliberately [17-22]. These studies are used to evaluate an analytical method's ability to measure an active ingredient and its degradation products without interference. Drug substances or drug products are exposed to acid, base, oxidizing agent, reducing agent, heat, and water to produce $10 \%-30 \%$ degradation of the drug substance. The degraded samples are then analyzed using the developed method to determine if there is any interference between the drug molecule and degradation compound(s). In current study conditions listed in Table 1 were applied.

2.5. Optimization by RSM. Experimental design through Response Surface Methodology was applied for the optimization and creating robust area of the mobile phase composition. A face centered Central Composite Design (CCD) with 2 factors and a total of 13 runs were selected for the optimization study.

The independent variables (factors) and their levels were selected from previous knowledge and preliminary experiments. Retention times of two drugs and resolution between their peaks were the responses. Factors, their limits, and responses with specific target were presented in Table 2.

The CCD design matrix and the obtained responses were subjected to multiple regression analysis and the resulting second-order polynomial function was used to correlate the independent variables and the responses. To verify the validity of the optimization procedure, a number of checkpoint analysis experiments were carried out in the design space and each experimental response was compared with the predicted one. 
TABLE 2: Independent variables (factor) and responses investigated in CCD design.

\begin{tabular}{lccccc}
\hline Independent variables (factors) & High $(+)$ & $\begin{array}{c}\text { Levels of factors } \\
\text { Medium }(0)\end{array}$ & Low $(-)$ & $\begin{array}{c}\text { Dependent variables } \\
\text { (responses) }\end{array}$ & Target of optimization \\
\hline Methanol $(\% \mathrm{v} / \mathrm{v})$ & 15 & 10 & 5 & Retention of PSU & $R_{t} \leq 3 \mathrm{~min}$ \\
Acetonitrile $(\% \mathrm{v} / \mathrm{v})$ & 60 & 40 & 20 & Retention of FEX & $3.5 \mathrm{~min} \leq R_{t} \leq 8 \mathrm{~min}$ \\
Buffer $(\% \mathrm{v} / \mathrm{v})$ & \multicolumn{2}{c}{ Quantity sufficient to $100 \%$} & Resolution $\left(R_{s}\right)$ & $6 \% \leq R_{s} \leq 15 \%$ \\
\hline
\end{tabular}

2.6. Statistical Analysis. The experimental design and regression analysis were carried out by Minitab 17 (Free Trial Version). The significance of independent variables and their interactions were tested by means of the analysis of variance (ANOVA). Various statistical indices, such as $P$ value, $F$ value, determination coefficient $\left(R^{2}\right)$, adjusted determination coefficient (adj $R^{2}$ ), and predicted determination coefficient (pred $R^{2}$ ), were used to assess the statistical significance and reliability of the quadratic models [23].

\section{Result and Discussion}

\subsection{Method Development}

3.1.1. Preliminary Screening. To determine the significant factors, their effects, and their levels, a preliminary screening and literature search were carried out. It was revealed from the screening that only acetonitrile in mobile phase in a range of 20 35\% separated the PSU and FEX with very low resolution and beyond $40 \%$ the peaks merged. On the other hand, only methanol in mobile phase, when used up to $50 \%$, tends to separate the molecules with very high resolution and take longer period to elute FEX (e.g., FEX eluted at $22 \mathrm{~min}$ at $40 \%$ methanol). From these observations it was clear that on $\mathrm{C}_{18}$ column there was a trend: acetonitrile tends to elute both the drugs quickly with low resolution whereas methanol tends to delay the elution of FEX. Consequently, it was stipulated that a mobile phase containing acetonitrile above $30 \%$ and methanol around $15 \%$ can effectively separate the drugs with good resolution. Therefore, in the optimization experiments, low and high level for acetonitrile were chosen as 20\% (v/v) and $60 \%(\mathrm{v} / \mathrm{v})$, respectively, and for methanol were $5 \%(\mathrm{v} / \mathrm{v})$ and $15 \%(\mathrm{v} / \mathrm{v})$. Other factors such as temperature, flow rate, and $\mathrm{pH}$ of the buffer were fixed based on knowledge as follows.

As a rule of thumb, $\mathrm{pH}$ of RP-HPLC mobile phase buffer should be 2 units above or below the pKa value of drug molecule $[7,8]$. The pKa of PSU and FEX is $9.8[24]$ and 4.25 , respectively [25]. We could not choose a $\mathrm{pH}$ above 9.8 as that higher $\mathrm{pH}$ is detrimental to column. We can choose 2 units below the pKa of FEX, but at that lower pH FEX remains undissociated and retention tends to be longer. In this case a pH around 6.8 seemed reasonable as it is 2 units above the pKa of FEX and 2 units below the pKa of PSU and both molecules remain dissociated which means faster elution. Consequently, $\mathrm{pH}$ was set at 6.8 . Flow rate was set at $1.0 \mathrm{~mL} / \mathrm{min}$.

Buffers are most effective at a $\mathrm{pH}$ close to their $\mathrm{pKa}$ value $(\mathrm{pKa} \pm 1)$. Phosphate buffer has a pKa of 7.1 which is close to the selected $\mathrm{pH}$ of mobile phase buffer. So, definitely our choice was phosphate buffer. Usual buffer concentration in RP-HPLC ranges from $10 \mathrm{mM}$ to $50 \mathrm{mM}$. In current study a buffer concentration from $10 \mathrm{mM}$ to $40 \mathrm{mM}$ revealed no major differences in retention and peak shape. Then we arbitrary chose the buffer concentration as $20 \mathrm{mM}$.

Also temperature variation between $22^{\circ} \mathrm{C}$ and $35^{\circ} \mathrm{C}$ showed no major variation in retention and peak shape. Therefore, room temperature was chosen. Chromatograms were best in appearance at $218 \mathrm{~nm}$.

3.1.2. Modeling for Optimization. A full factorial design with 13 experimental conditions was performed including 9 conditions defined by the design of experiments with 5 repetitions at the center of the domain. A chromatogram was recorded for each of these conditions. Target was to minimize the retention time of both drugs and at the same time maximize the resolution. The runs and corresponding responses were depicted in Table 3.

As it was observed, at all combinations, the retention of PSU remained below 3.5 minutes but that of FEX varied widely ranging from as low as 3.3 minutes to as high as 37.6 minutes. Resolutions between two peaks were found in a range of $0.75 \%$ to $38.16 \%$ which was a clear indication that the model could be effectively optimized to achieve our goal.

The ANOVA was conducted to test the significance and validity of the quadratic models for the experimental data, results of which were presented in Table 4 . The significance of each model is evident from Fisher's ratio ( $F$-value) and $P$ value. $F$-value for model PSU, model FEX, and model $R_{s}$ were found to be $98.06,74.95$, and 48.87 , respectively, and $P$ value for all three models was found to be 0.0001 . The high $F$-value and $P$ value $<0.05$ for each model indicated that the models were significant and can be used to navigate the design space.

The quality of the obtained polynomial regression was assessed by the determination coefficient $\left(R^{2}\right)$, adjusted determination coefficient (adj $R^{2}$ ), predicted determination coefficient (pred $R^{2}$ ), the graph residues, and the adequacy between the retention times predicted by the model and those observed.

In the current experiments, $R^{2}$ and adjusted $R^{2}$ were high for all three models. Pred $R^{2}$ were in reasonable agreement with adjusted $R^{2}$ (difference is less than 20\%). Table 5 represented the model summery.

The normal probability plots illustrated in Figure 1 showed that errors are normally distributed and independent of each other and are homogenous. The residual plots showed random distribution of residuals without any trend, indicating good prediction of maximum response along with the 
TABLE 3: CCD design matrix with two independent variables and observed responses.

\begin{tabular}{|c|c|c|c|c|c|c|c|}
\hline Std order & Run order & Blocks & $\mathrm{MeOH}(\% \mathrm{~V} / \mathrm{V})$ & $\mathrm{ACN}(\% \mathrm{~V} / \mathrm{V})$ & $R_{t}$ of PSU (min) & $R_{t}$ of FEX (min) & $R_{s}(\%)$ \\
\hline 2 & 1 & 1 & 15 & 20 & 3.30 & 37.6 & 38.16 \\
\hline 13 & 2 & 1 & 10 & 40 & 2.50 & 3.40 & 4.55 \\
\hline 11 & 3 & 1 & 10 & 40 & 2.40 & 3.30 & 4.5 \\
\hline 8 & 4 & 1 & 10 & 60 & 2.60 & 4.10 & 6.27 \\
\hline 9 & 5 & 1 & 10 & 40 & 2.50 & 3.50 & 4.7 \\
\hline 3 & 6 & 1 & 5 & 60 & 2.70 & 2.98 & 0.75 \\
\hline 5 & 7 & 1 & 5 & 40 & 2.54 & 3.26 & 3.34 \\
\hline 12 & 8 & 1 & 10 & 40 & 2.50 & 3.40 & 4.55 \\
\hline 4 & 9 & 1 & 15 & 60 & 2.70 & 3.80 & 5.2 \\
\hline 7 & 10 & 1 & 10 & 20 & 3.00 & 20.2 & 29.4 \\
\hline 10 & 11 & 1 & 10 & 40 & 2.50 & 3.40 & 4.55 \\
\hline 6 & 12 & 1 & 15 & 40 & 2.70 & 4.50 & 8.57 \\
\hline 1 & 13 & 1 & 5 & 20 & 3.10 & 35.0 & 36.25 \\
\hline
\end{tabular}

TABLE 4: ANOVA for $2^{3}$ full factorial design: responses are retention times of the drugs (PSU and FEX) and resolution $\left(R_{s}\right)$.

\begin{tabular}{|c|c|c|c|c|c|c|c|c|}
\hline \multirow{2}{*}{ Source } & \multirow{2}{*}{ DF } & \multicolumn{3}{|c|}{$F$-value } & \multicolumn{3}{|c|}{$P$ value } & \multirow{2}{*}{ Remark } \\
\hline & & PSU & FEX & $R_{s}$ & PSU & FEX & $R_{s}$ & \\
\hline Model & 5 & 98.06 & 74.95 & 48.87 & 0.0001 & 0.0001 & 0.0001 & Significant \\
\hline Linear & 2 & 96.72 & 119.5 & 88.76 & 0.0001 & 0.0001 & 0.0001 & Significant \\
\hline Square & 2 & 145.7 & 67.27 & 32.03 & 0.0001 & 0.0001 & 0.0001 & Significant \\
\hline Interaction & 1 & 5.55 & 4.20 & 2.80 & 0.051 & 0.031 & 0.013 & Significant \\
\hline Lack-of-fit & 3 & 0.77 & 0.324 & 0.490 & 0.569 & 0.295 & 0.120 & Not significant \\
\hline
\end{tabular}

TABLE 5: Model summary for transformed responses.

\begin{tabular}{lcccc}
\hline Model & $R^{2}$ & $\operatorname{adj} R^{2}$ & pred $R^{2}$ & adj $R^{2}-\operatorname{pred~} R^{2}$ \\
\hline PSU versus ACN, $\mathrm{MeOH}$ & $98.59 \%$ & $97.59 \%$ & $93.49 \%$ & $4.10 \%$ \\
FEX versus ACN, $\mathrm{MeOH}$ & $98.17 \%$ & $96.86 \%$ & $82.33 \%$ & $14.53 \%$ \\
$R_{s}$ versus ACN, $\mathrm{MeOH}$ & $97.22 \%$ & $95.23 \%$ & $82.56 \%$ & $12.67 \%$ \\
\hline
\end{tabular}

constant variance. These data further confirm the reliability of the quadratic models and their suitability for the optimization of mobile phase.

\subsubsection{Design Space and Condition for Optimal Separation.} The standardized effects of the independent factors and their interactions on responses were visualized by threedimensional response surface graph and two-dimensional contour plots in Figure 2.

Using the data obtained by experimental conditions and corresponding responses, overlay contour plots were constructed to determine the design space and find out the optimal composition of mobile phase. In this case target was to minimize the retention time of both drug and at the same time maximize the resolution. Minimum and maximum limits of our desired responses were set as depicted in Figure 3. The white region in the figure indicated the design space for both drugs. The composite desirability of the system at different conditions within the design space was calculated, and the conditions at which the composite desirability of system was more than 0.80 were selected as the optimal conditions. Four different points within the feasible region were primarily chosen for further investigation (Figure 3). The composite desirability of those points was determined. At all four compositions, separations were in agreement with the prediction. Finally we chose the method as "Buffer : Acetonitrile : Methanol = 50:36:14 (\% v/v)" due mainly to two reason: firstly, the composite desirability was found highest (0.97) at this point, and secondly, at this point tailing of PSU was at minimum (1.6).

\subsection{Method Validation}

3.2.1. Specificity. By visual inspection of the standard chromatogram, assay sample chromatogram, and dissolution sample chromatogram, no interference with any other peak from impurities or excipients was found. Moreover, peak purity indices in all cases were checked and found more than 0.9998 which indicated specificity of this method for the simultaneous separation of the drug molecules. Figure 4 showed the chromatograms.

3.2.2. Solution Stability. Two vials, one containing the working standard and another the tablet sample at nominal 

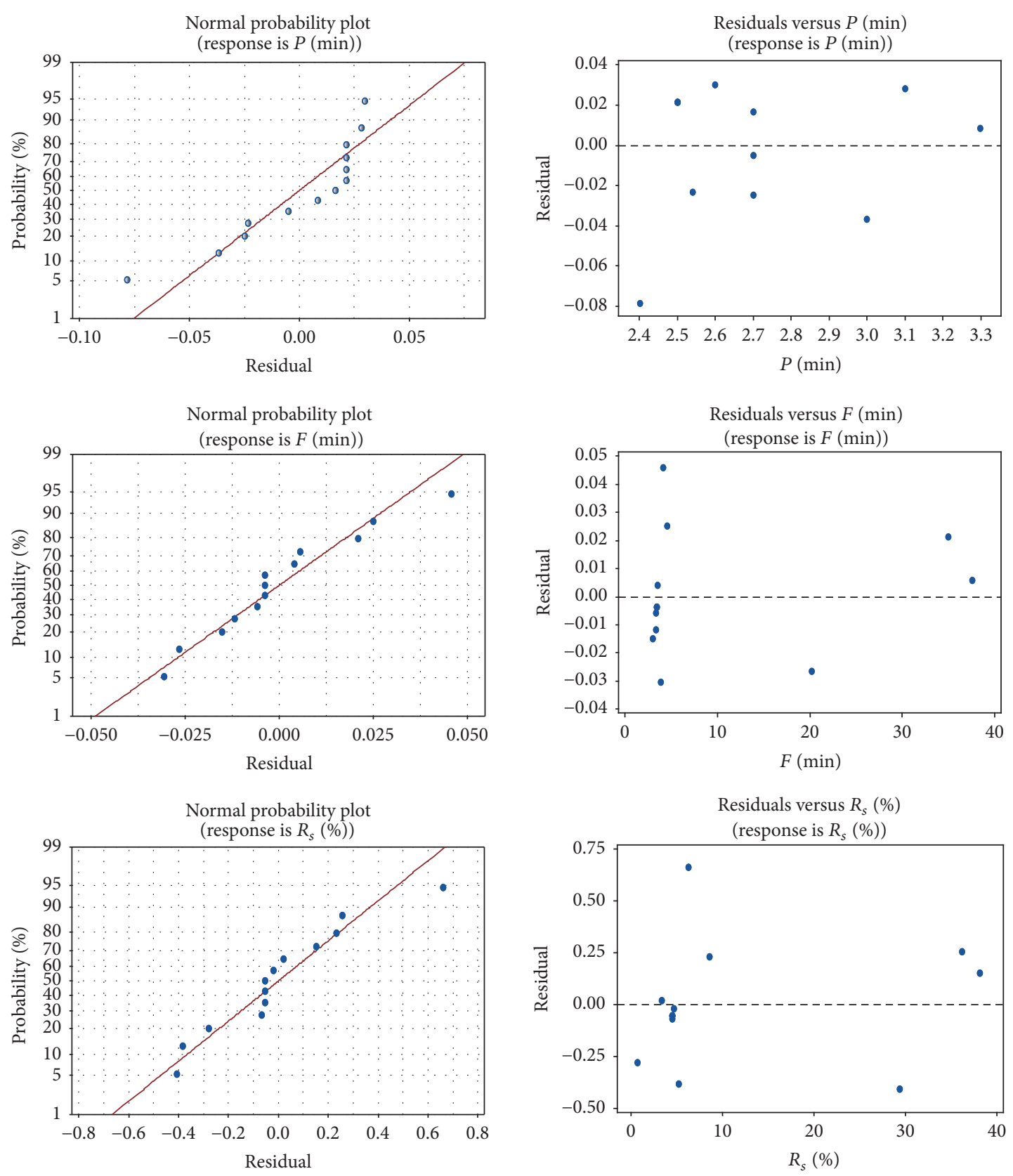

FIGURE 1: Normal probability plots of residuals and the factor versus residual plots of the proposed quadratic model.

concentration, were kept at room temperature and were tested at 0th hour, 24th hour, and 48th hour. Then the peak areas were compared with that of 0th hour. No significant changes in area were observed. \% RSD was found below $1.5 \%$ which indicated the stability of drugs in diluting solvent.

3.2.3. Linearity, Working Range, and Accuracy. In the current validation procedure nominal standard concentration was $30 \mu \mathrm{g} / \mathrm{mL}$ PSU and $15 \mu \mathrm{g} / \mathrm{mL}$ FEX. We made $50 \%, 80 \%$, $90 \%, 110 \%, 120 \%, 150 \%$, and $200 \%$ of the nominal standard solution and the peak area was plotted against corresponding concentration. The regression coefficient $\left(r^{2}\right)$ was 0.999 for both drugs, which indicated the acceptable fit of the data to the regression line. The working range was derived from the linearity curve and it was found as $15 \mu \mathrm{g} / \mathrm{mL} \sim 60 \mu \mathrm{g} / \mathrm{mL}$ for PSU and $7.5 \mu \mathrm{g} / \mathrm{mL} \sim 30 \mu \mathrm{g} / \mathrm{mL}$ for FEX. The regression equations were as follows:

$$
\begin{aligned}
& \text { PSU: } y=23541 x-11897, \\
& \text { FEX: } y=44546 x-430.41 .
\end{aligned}
$$

Percent recoveries for all concentrations were found within $100 \% \pm 2 \%$ and the \% RSD of recoveries was found very low indicating accuracy of the method for simultaneous estimation of the drugs. 
Surface plot of $P(\mathrm{~min})$ versus $\mathrm{ACN}(\% \mathrm{~V} / \mathrm{V}), \mathrm{MeOH}(\% \mathrm{~V} / \mathrm{V})$

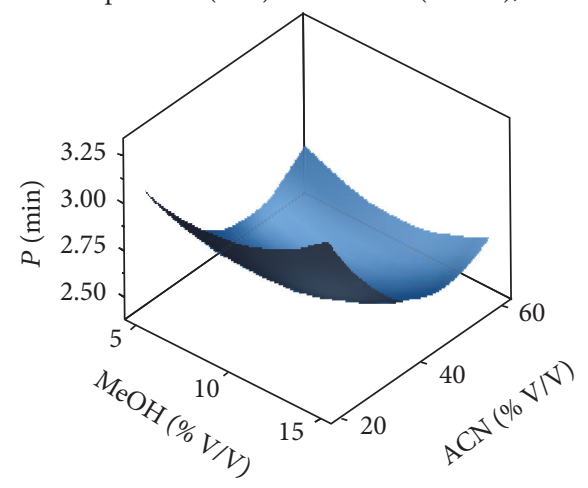

Surface plot of $F(\mathrm{~min})$ versus $\mathrm{ACN}(\% \mathrm{~V} / \mathrm{V}), \mathrm{MeOH}(\% \mathrm{~V} / \mathrm{V})$

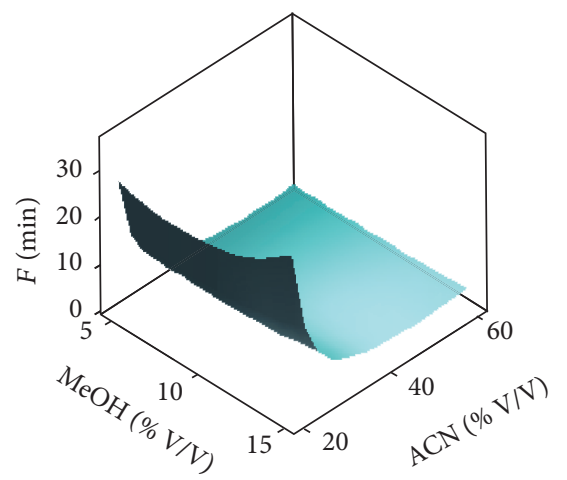

Surface plot of $R_{s}(\%)$ versus $\mathrm{ACN}(\% \mathrm{~V} / \mathrm{V}), \mathrm{MeOH}(\% \mathrm{~V} / \mathrm{V})$

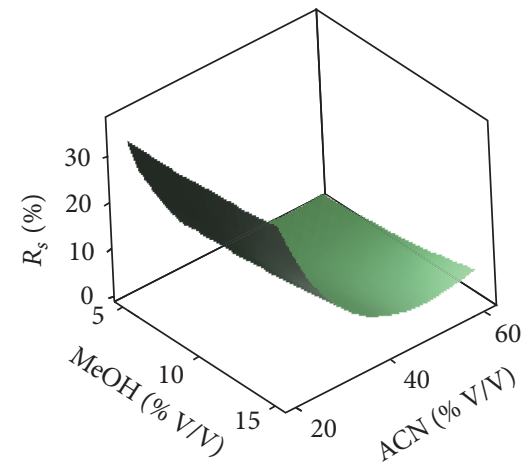

Contour plot of $P(\mathrm{~min})$ versus $\mathrm{ACN}(\% \mathrm{~V} / \mathrm{V}), \mathrm{MeOH}(\% \mathrm{~V} / \mathrm{V})$

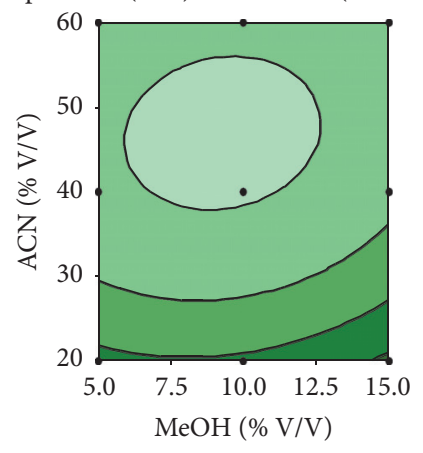

$$
\begin{aligned}
& P(\min )
\end{aligned}
$$

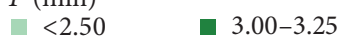

$$
\begin{aligned}
& \text { - } 2.50-2.75 \\
& \text { - 2.75-3.00 }
\end{aligned}
$$

Contour plot of $F(\mathrm{~min})$ versus $\mathrm{ACN}(\% \mathrm{~V} / \mathrm{V}), \mathrm{MeOH}(\% \mathrm{~V} / \mathrm{V})$

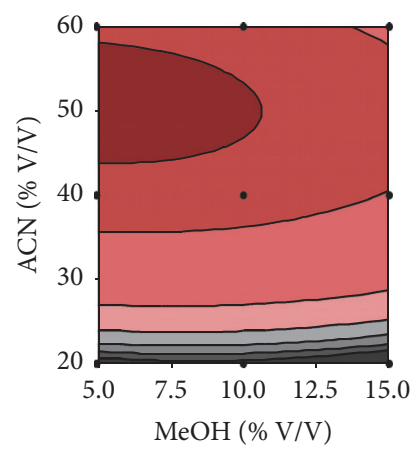

$$
\begin{aligned}
& F \text { (min) } \\
& \text { - }<3 \text { - 12-16 } \\
& \text { - 3-4 16-20 } \\
& \text { ㅁ 4-8 } 20-24 \\
& \text { - } 8-12 \text { a }>24
\end{aligned}
$$

Contour plot of $R_{s}(\%)$ versus $\mathrm{ACN}(\% \mathrm{~V} / \mathrm{V}), \mathrm{MeOH}(\% \mathrm{~V} / \mathrm{V})$

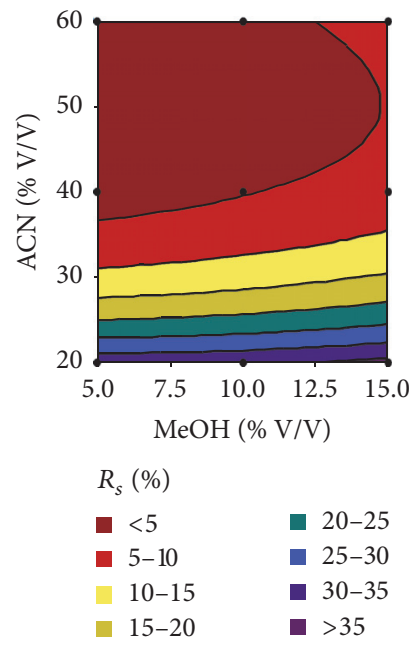

FIGURE 2: Three-dimensional response surface graphs and individual contour plots. 
Contour plot of $R_{s}(\%), F(\mathrm{~min}), P(\mathrm{~min})$

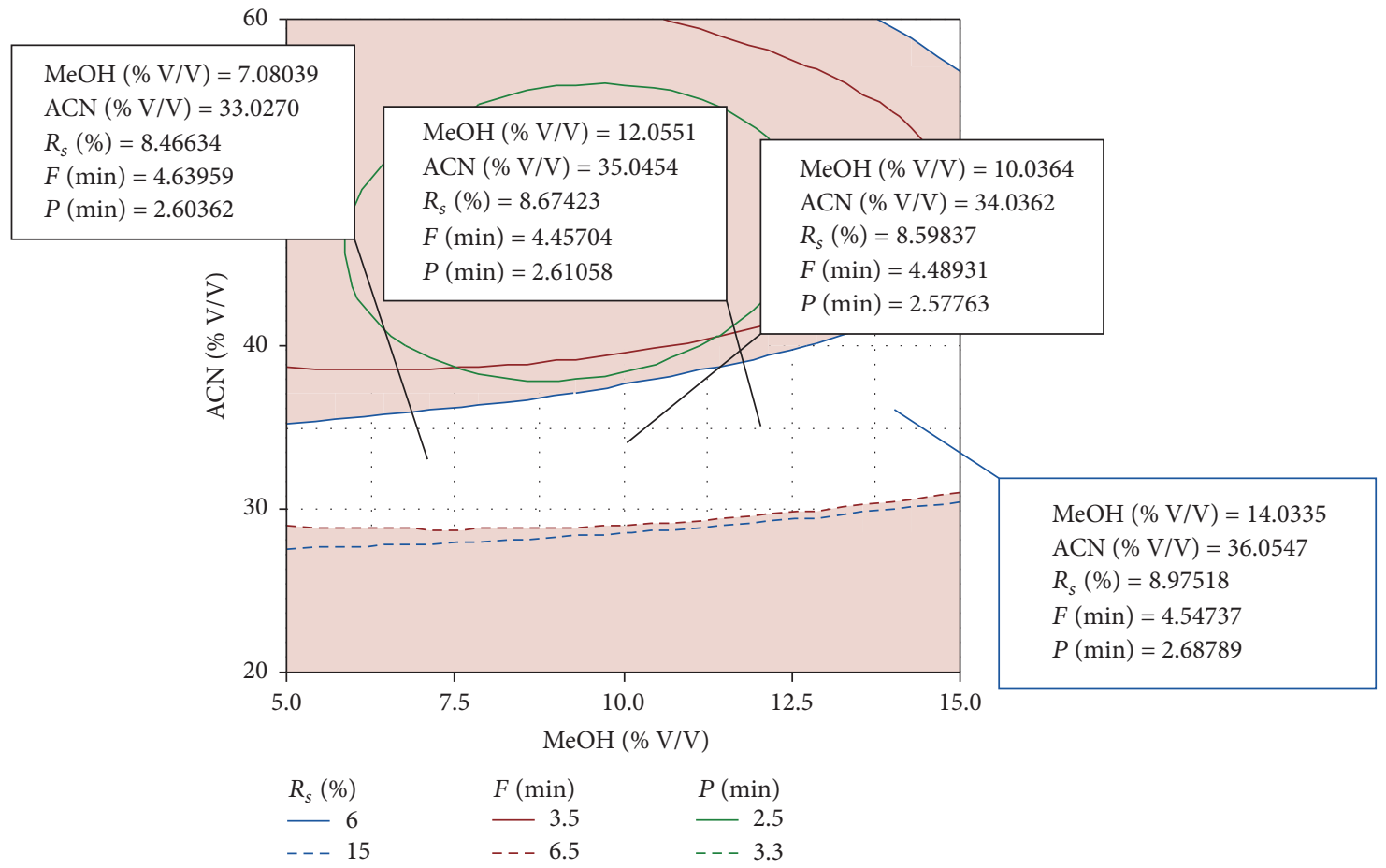

FIGURE 3: Overlaid contour plot of the responses and the design space: $F(\mathrm{~min})$ and $P(\mathrm{~min})$ are the retention time of FEX and PSU in minute. Solid lines indicated the desired lower limit and broken lines indicated the desired upper limit of each response.

TABLE 6: Summary of precision.

\begin{tabular}{|c|c|c|c|c|c|c|}
\hline \multirow{2}{*}{ Run order } & \multicolumn{3}{|c|}{ PSU } & \multicolumn{3}{|c|}{ FEX } \\
\hline & $50 \%$ & $100 \%$ & $150 \%$ & $50 \%$ & $100 \%$ & $150 \%$ \\
\hline HPLC-1, day 1 & 100.05 & 99.98 & 100.02 & 99.99 & 99.98 & 99.98 \\
\hline HPLC-1, day 2 & 99.99 & 99.92 & 100.07 & 99.99 & 100.01 & 100.51 \\
\hline HPLC-2, day 1 & 99.94 & 100.10 & 100.56 & 99.98 & 100.10 & 99.97 \\
\hline HPLC-2, day 2 & 100.01 & 99.90 & 100.10 & 99.99 & 100.06 & 99.94 \\
\hline$\%$ RSD of total variation & 0.0457 & 0.090 & 0.2500 & 0.005 & 0.0531 & 0.2735 \\
\hline \multirow{2}{*}{ \% RSD of HPLC ${ }^{\mathrm{a}}$ system } & 0.0777 & 0.0849 & 0.3807 & 0.0071 & 0.0849 & 0.0071 \\
\hline & 0.0141 & 0.0141 & 0.0211 & 0.0010 & 0.0354 & 0.4021 \\
\hline \multirow{2}{*}{$\%$ RSD of interday ${ }^{\mathrm{b}}$ assay precision } & 0.0424 & 0.0424 & 0.0353 & 0.00 & 0.0212 & 0.3738 \\
\hline & 0.0495 & 0.1414 & 0.3241 & 0.007 & 0.0283 & 0.0212 \\
\hline
\end{tabular}

${ }^{a}$ Upper row for HPLC variation on day 1 and lower row for HPLC variation on day 2

${ }^{\mathrm{b}}$ Upper row for interday variation on HPLC-1 and lower row for interday variation on HPLC-2.

3.2.4. Precision. In the present study, three different solutions were prepared with known added amounts of drugs, namely, $50 \%, 100 \%$, and $150 \%$, of nominal concentration and injected in triplicate. Percent recoveries (concentrations) were calculated using regression equation. The result of precision studies was shown in Table 6. The low \% RSD of total variation, interday variation, and interinstrument variation clearly evinced that the method was precise within the desired recovery range.

3.2.5. Forced Degradation Study. It is evident from the forced degradation study that both PSU and FEX are fairly stable in acidic condition. This finding also justifies the choice of dissolution medium of this combination formulation as $0.001 \mathrm{~N} \mathrm{HCl}$ where PSU remains 12 hours during dissolution testing. Their stability in acidic medium may be accounted by the fact that both of the drugs exist in hydrochloric acid salt form which tends to remain undissociated in acidic media. So it can be stipulated from this data that PSU and FEX are essentially stable in the stomach $\mathrm{pH}$. On the other hand, both drugs are fairly sensitive to basic medium which promotes their dissociation and consequent degradation reaction. These findings provide important information of selection of the $\mathrm{pH}$ of any dosage form containing PSU and/or 


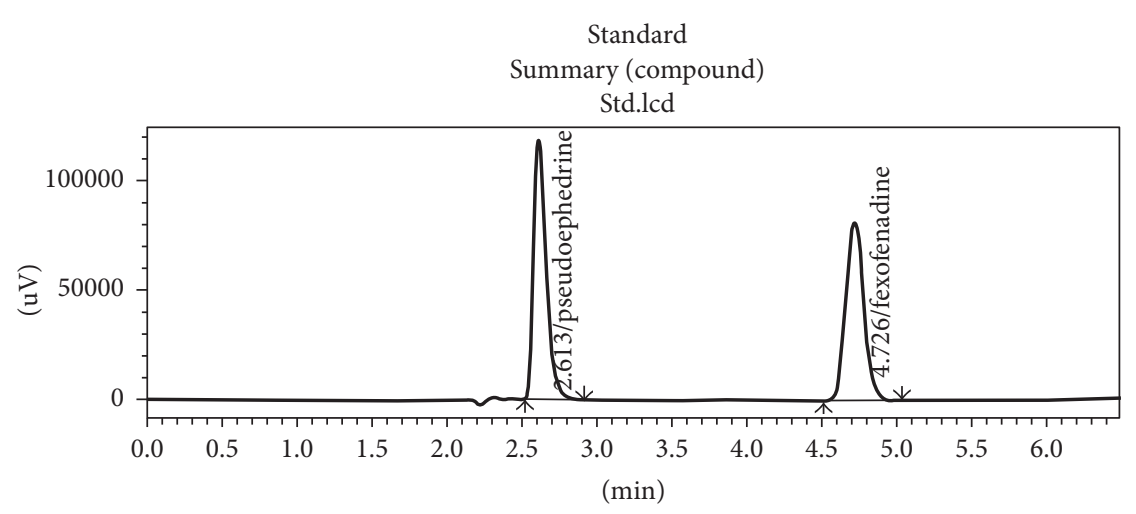

(a)

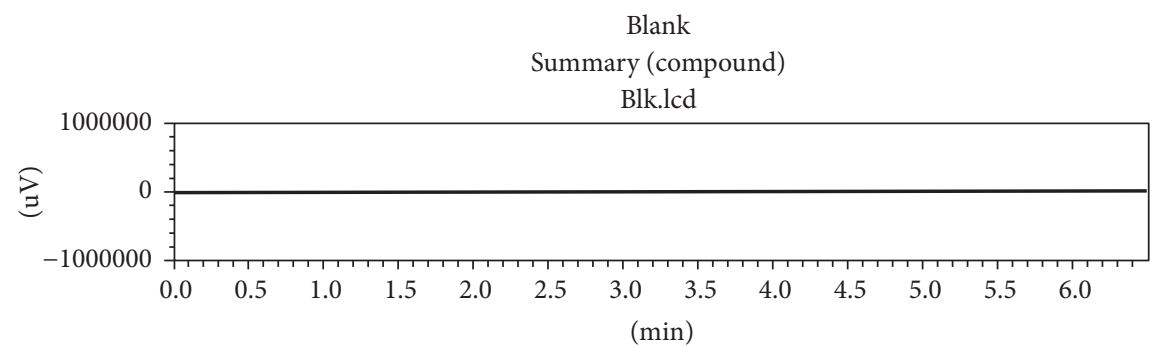

(b)

Sample assay

Summary (compound)

Sample.lcd

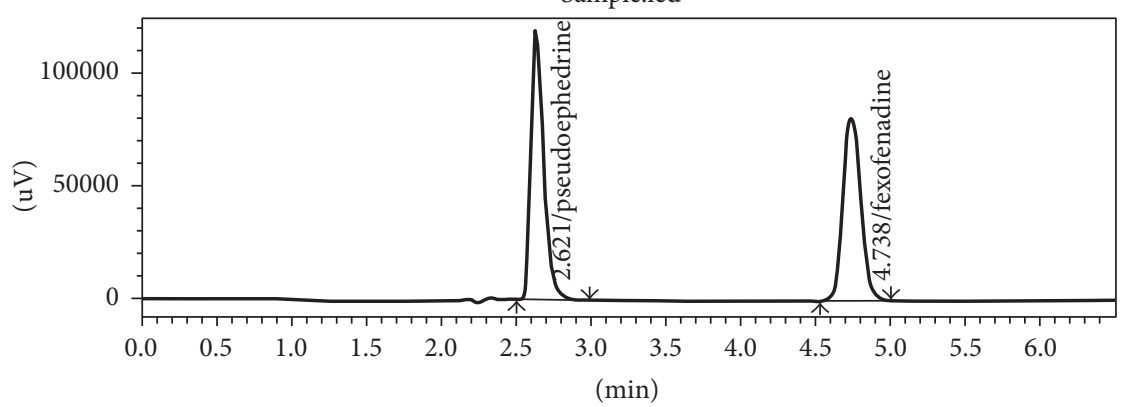

(c)

Sample dissolution

Summary (compound) dissolution.lcd

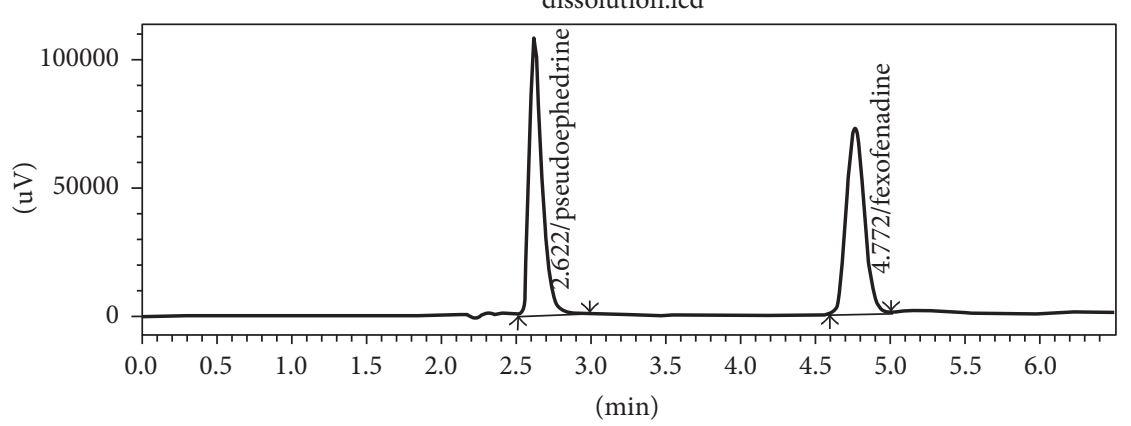

(d)

FIgURE 4: Chromatogram of (a) standard solution, (b) blank, (c) assay sample of tablet, and (d) dissolution sample. 
TABLE 7: Result of forced degradation study.

\begin{tabular}{lcccc}
\hline Drug & \% loss in acidic media & \% loss in basic media & \% loss in oxidation & \% loss in reduction \\
\hline \% loss of PSU & -0.90908 & -61.9284 & -100 & -14.4364 \\
\% loss of FEX & +0.293962 & -84.6267 & -93.7939 & -19.5013 \\
\hline
\end{tabular}

TABLE 8: System suitability study.

\begin{tabular}{|c|c|c|c|}
\hline Parameter & PSU & FEX & Limit [14-16] \\
\hline$\%$ RSD of area ${ }^{a}$ & 0.592 & 0.484 & $\% \mathrm{RSD} \leq 2 \%$ \\
\hline$\%$ RSD of retention ${ }^{a}$ & 0.192 & 0.211 & $\% \mathrm{RSD} \leq 2 \%$ \\
\hline Tailing factor $\left(T_{f}\right)$ & 1.626 & 1.150 & $\mathrm{USP} T_{f} \leq 2.0$ \\
\hline Resolution $\left(R_{s}\right)$ & - & 10.94 & $R_{s} \geq 2.0$ \\
\hline $\mathrm{LOD}^{\mathrm{b}}$ & $0.018 \mu \mathrm{g} / \mathrm{mL}$ & $0.013 \mu \mathrm{g} / \mathrm{mL}$ & $S / N=3: 1$ \\
\hline $\mathrm{LOQ}^{\mathrm{b}}$ & $0.055 \mu \mathrm{g} / \mathrm{mL}$ & $0.041 \mu \mathrm{g} / \mathrm{mL}$ & $S / N=10: 1$ \\
\hline
\end{tabular}

${ }^{\mathrm{a}} n=6 .{ }^{\mathrm{b}}$ Based on visual detection.

TABLE 9: Result of robustness study.

\begin{tabular}{|c|c|c|c|c|c|c|c|}
\hline \multirow{2}{*}{ Parameter } & \multirow{2}{*}{ Changed value } & \multicolumn{2}{|c|}{ Retention } & \multicolumn{2}{|c|}{ Tailing factor } & \multicolumn{2}{|c|}{ Peak purity } \\
\hline & & PSU & FEX & PSU & FEX & PSU & FEX \\
\hline \multirow{2}{*}{$\mathrm{pH} \pm 0.4$} & 7.2 & 3.0 & 4.6 & 1.63 & 1.15 & 0.999 & 1.000 \\
\hline & 6.4 & 2.5 & 4.6 & 1.62 & 1.14 & 0.999 & 1.000 \\
\hline \multirow{2}{*}{ Flow \pm 0.5} & 1.5 & 1.8 & 3.2 & 1.48 & 1.10 & 1.000 & 1.000 \\
\hline & 0.5 & 4.8 & 7.0 & 1.71 & 1.21 & 0.999 & 0.999 \\
\hline \multirow{2}{*}{ Buffer conc. $\pm 10 \mathrm{mM}$} & $30 \mathrm{mM}$ & 2.8 & 4.9 & 1.59 & 1.13 & 1.000 & 0.999 \\
\hline & $10 \mathrm{mM}$ & 2.6 & 4.4 & 1.61 & 1.21 & 1.000 & 1.000 \\
\hline Zorbax Eclipse XDB (Double Encapped) & Nonpolar & 2.8 & 4.8 & 1.68 & 1.10 & 1.000 & 0.999 \\
\hline $\begin{array}{l}\text { ProntoSIL C18-EPS (with amide } \\
\text { embedded group) }\end{array}$ & Polar & 3.5 & 4.1 & 1.36 & 1.31 & 0.999 & 1.000 \\
\hline
\end{tabular}

FEX. These drugs have shown highest degradation propensity towards oxidizing agents. One very conspicuous change is that PSU is absolutely sensitive to oxidizing agents as no peak of this drug has been detected, even at LOD level, in the oxidized sample. Therefore, formulator should consider the addition of an antioxidant in the formula of any dosage form containing PSU and FEX. Both drugs are moderately sensitive to reducing agent. No degradation was found under heating condition. All these data obtained in this degradation study can be very precious for the formulator intending to develop a stable formula of PSU and FEX either in solid dosage form or in liquid dosage form. Results were summarized in Table 7.

3.2.6. System Suitability. All the system suitability parameters met the compendial specification. Results were presented in Table 8.

3.2.7. Robustness. In our robustness study, flow rate, buffer concentration, $\mathrm{pH}$ of mobile phase, and column variation were studied. Such responses as retention, tailing factor, and peak purity were in acceptable ranges. Results were shown in Table 9.

\section{Applicability of Method}

The developed method was applied to assay the quantity of PSU and FEX in the two local brands of extended tablet dosage form of these drugs combination, namely, Ritch Plus (ACI Limited) and Fexo ${ }^{\mathrm{TM}}$ Plus (Square Pharmaceuticals Limited). Assay result was compared with that determined by the method outlined in USP 2015. In the same way dissolution rates of these two brands were evaluated by current methods and USP method. In all cases similar results were found.

\section{Conclusion}

So, a simple, rapid, and stability-indicating reversed phase HPLC method was developed for simultaneous estimation of PSU and FEX by applying design of experiments and statistical analysis. Design space was successfully created and optimum condition was derived within the robust area. To the best of our knowledge, this method may be considered superior than any other methods stated elsewhere. Use of same column and mobile phase in both assay and dissolution rate measurement can be deemed as the special attribute of this new method. All the validation parameters satisfactorily 
met the characteristics of a precise, accurate, and robust RP-HPLC method. Therefore, this method can be applied in research laboratory and in pharmaceutical industries for routine analysis of PSU and FEX in their combined extended release tablets.

\section{Competing Interests}

Authors declare no conflict of interests.

\section{Authors' Contributions}

The study was carried out in collaboration among all the authors. The idea was developed by R. Kayesh. A. S. M. M. Sarker prepared the validation protocol. Analyses, design, and optimizations were done by R. Kayesh and M. S. Jahan. R. Kayesh wrote the first draft of the manuscript and arranged the references which were edited and finalized by M. S. Jahan and M. Z. Sultan.

\section{Acknowledgments}

Authors are very grateful to the Director of Quality Assurance of ACI Limited for providing the active ingredients and reagents. Authors are also very thankful to Dr. Md. Zakir Sultan for his kind cooperation and generous support in running this research work in his lab.

\section{References}

[1] S. C. Sweetman, Martindale. The Complete Drug Reference, 35th edition, 2006.

[2] J. G. Hardman and L. E. Limbird, Goodman \& Gilman's The Pharmacology Basis of Therapeutics, McGraw-Hill, New York, NY, USA, 10th edition, 2001.

[3] http://dailymed.nlm.nih.gov/dailymed/drugInfo.cfm?setid= f9a15b63-f48e-4802-bd4d-53fdb65ca6a2.

[4] http://www.rxlist.com/allegra-d-drug.htm.

[5] M. Induri, A. Fathima, R. M. Bhagavan, and P. Y. Rajendra, "Simultaneous quantification of pseudoephedrine hydrochloride and fexofenadine hydrochloride in tablets by liquid chromatography," Jordan Journal of Pharmaceutical Sciences, vol. 6, no. 3, pp. 323-329, 2013.

[6] S. Karakuş, I. Küçükgüzel, and Ş. G. Küçükgüzel, "Development and validation of a rapid RP-HPLC method for the determination of cetirizine or fexofenadine with pseudoephedrine in binary pharmaceutical dosage forms," Journal of Pharmaceutical and Biomedical Analysis, vol. 46, no. 2, pp. 295-302, 2008.

[7] S. Ahuja and S. Scypinski, Handbook of Modern Pharmaceutical Analysis, vol. III, 2001.

[8] L. Ohannesian and A. J. Streeter, Handbook of Pharmaceutical Analysis, CRC Press, 2005.

[9] E. A. Sharma and N. J. Shah, "Stability indicating RP-HPLC method development and validation for pseudoephedrine hydrochloride and fexofenadine hydrochloride in tablet dosage form," International Journal of Pharmaceutical Sciences and Research, vol. 6, no. 12, pp. 5245-5252, 2015.

[10] https://medlineplus.gov/druginfo/meds/a601113.html.

[11] https://en.wikipedia.org/wiki/Docusate\#Other_uses.
[12] C. R. Raymond, J. S. Paul, and E. Q. Marian, Handbook of Pharmaceutical Excipients, 6th edition, 2016.

[13] The United States Pharmacopeia, USP38-NF33, vol. 2, 2015.

[14] International Conference on Harmonization (ICH), Q2B: Validation of Analytical Procedures: Methodology, 1997.

[15] Food and Drug Administration, "Validation and verification guidance for human drug analytical methods (Appendix 1)," Document No. ORA-LAB.5.4.5, version no.: 1.6, 2003, http://www.fda.gov/downloads/ScienceResearch/FieldScience/ UCM092147.pdf.

[16] United States Pharmacopoeia 38-National Formulary 33 (USP 38-NF 33), Section (1225), United States Pharmacopeial Convention, Rockville, Md, USA, 2007.

[17] "ICH guidelines Q1A (R2). Stability Testing of New Drug Substances and Products (revision 2)," November 2003.

[18] D. W. Reynolds, K. L. Facchine, J. F. Mullaney, K. M. Alsante, T. D. Hatajik, and M. G. Motto, "Available guidance and best practices for conducting forced degradation studies," Pharmaceutical Technology, vol. 26, no. 2, pp. 48-56, 2002.

[19] N. G. Shinde, B. N. Bangar, S. M. Deshmukh, S. P. Sulake, and D. P. Sherekar, "Pharmaceutical forced degradation studies with regulatory consideration," Asian Journal of Research in Pharmaceutical Sciences, vol. 3, no. 4, pp. 178-188, 2013.

[20] T. Rawat and I. P. Pandey, "Forced degradation studies for drug substances and drug products- scientific and regulatory considerations," Journal of Pharmaceutical Sciences and Research, vol. 7, no. 5, pp. 238-241, 2015.

[21] B. R. Matthews, "Regulatory aspects of stability testing in Europe," Drug Development and Industrial Pharmacy, vol. 25, no. 7, pp. 831-856, 1999.

[22] R. Nageswara Rao and V. Nagaraju, "An overview of the recent trends in development of HPLC methods for determination of impurities in drugs," Journal of Pharmaceutical and Biomedical Analysis, vol. 33, no. 3, pp. 335-377, 2003.

[23] D. C. Montgomery, Design and Analysis of Experiments, 5th edition, 2004.

[24] M. Yolanda, S. Martono, and A. Rohman, "Validation of an ion-pair high performance liquid chromatography for the determination of pseudoephedrine $\mathrm{HCl}$, guaifenesin and dexchlorpheniramine maleat in cough and cold medicines," International Journal of Pharmaceutical and Clinical Research, vol. 8, no. 5, pp. 476-480, 2016.

[25] https://pubchem.ncbi.nlm.nih.gov/compound/fexofenadine\# section $=\log P$. 

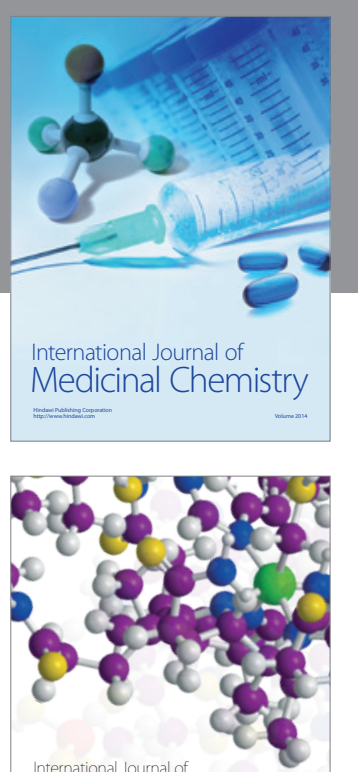

Carbohydrate Chemistry

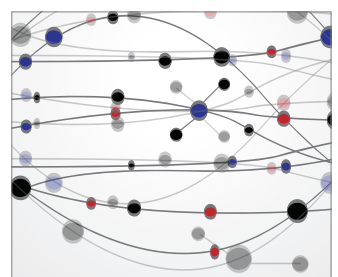

The Scientific World Journal
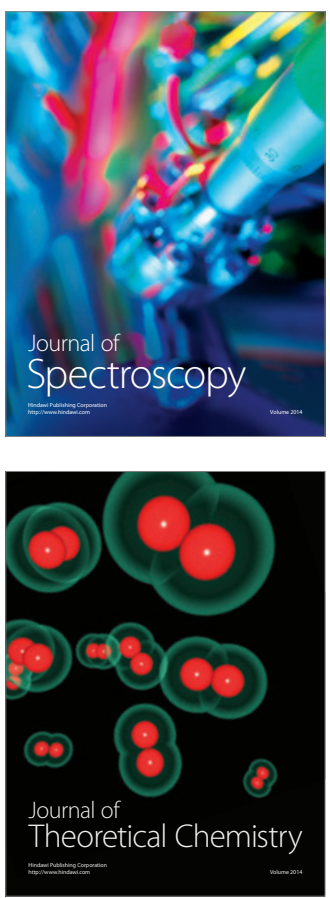
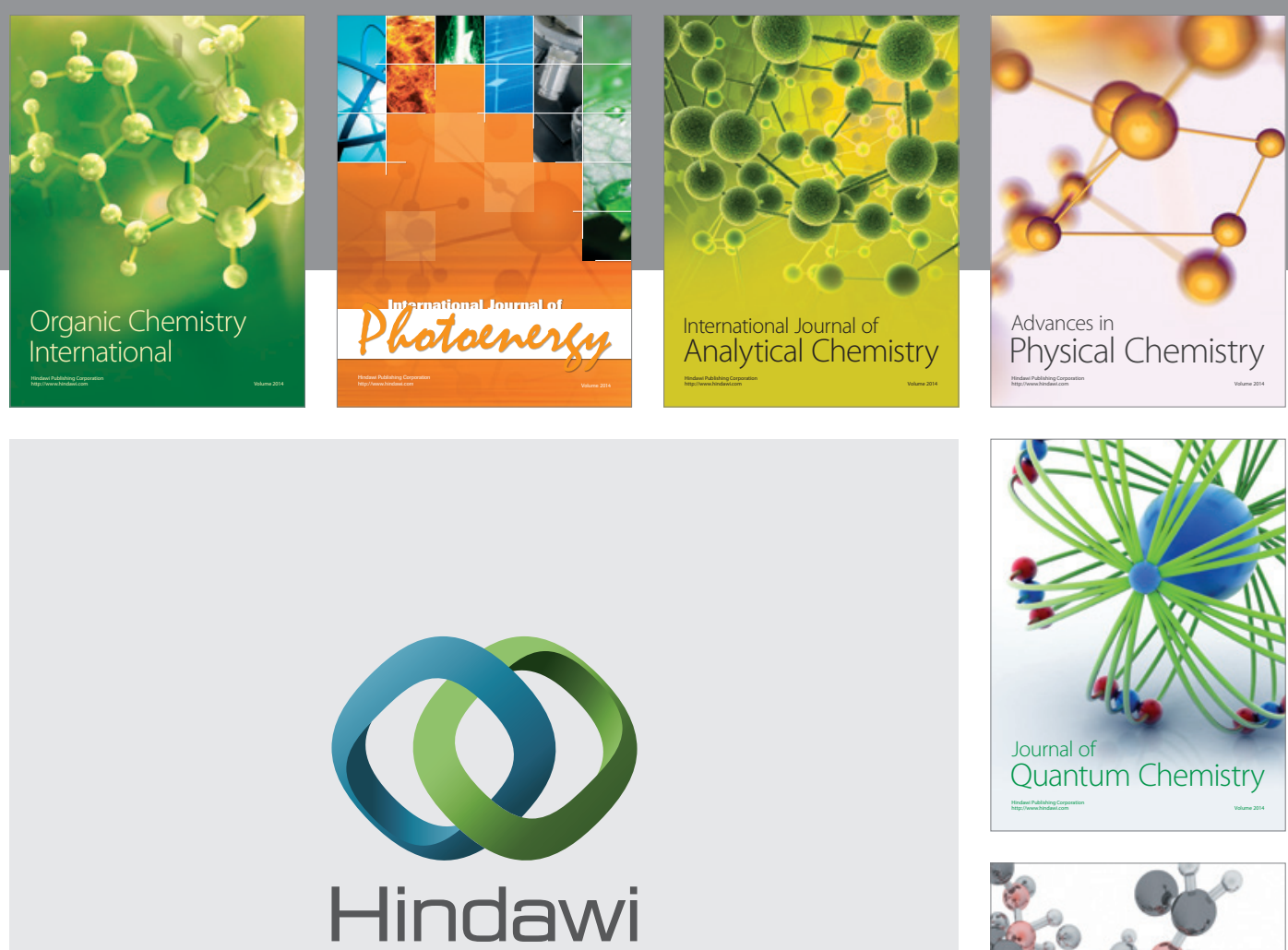

Submit your manuscripts at

https://www.hindawi.com

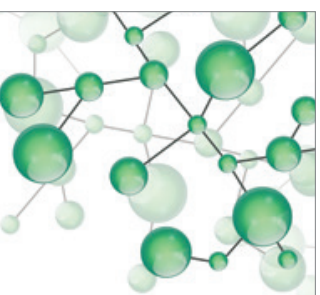

International Journal of

Inorganic Chemistry
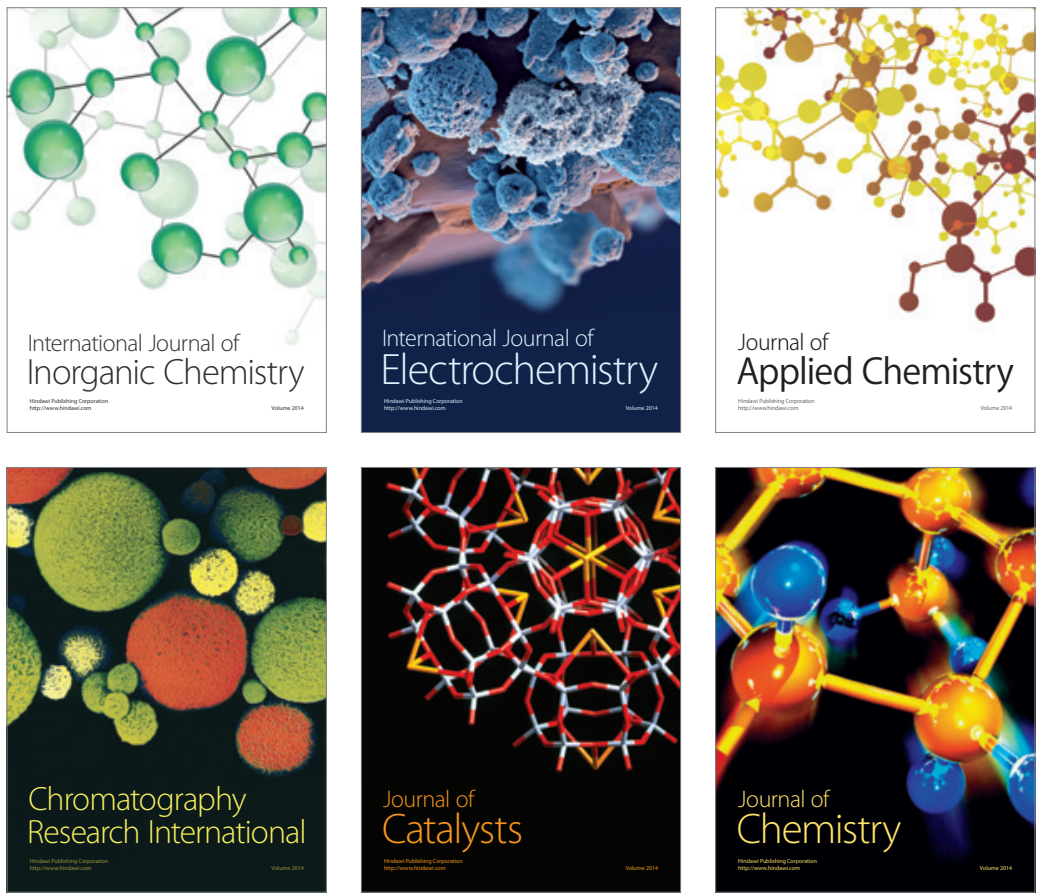

Journal of

Applied Chemistry
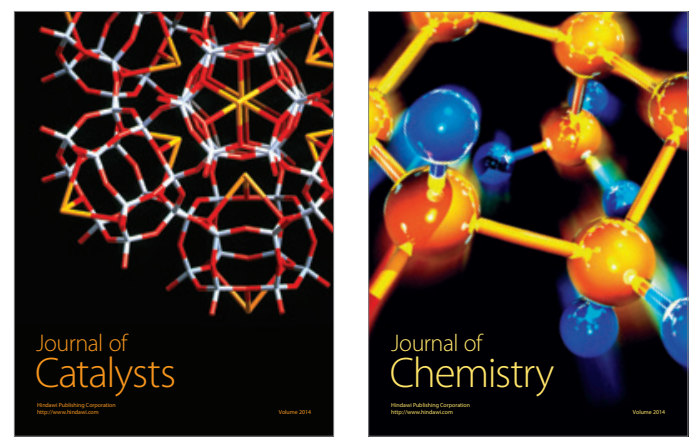
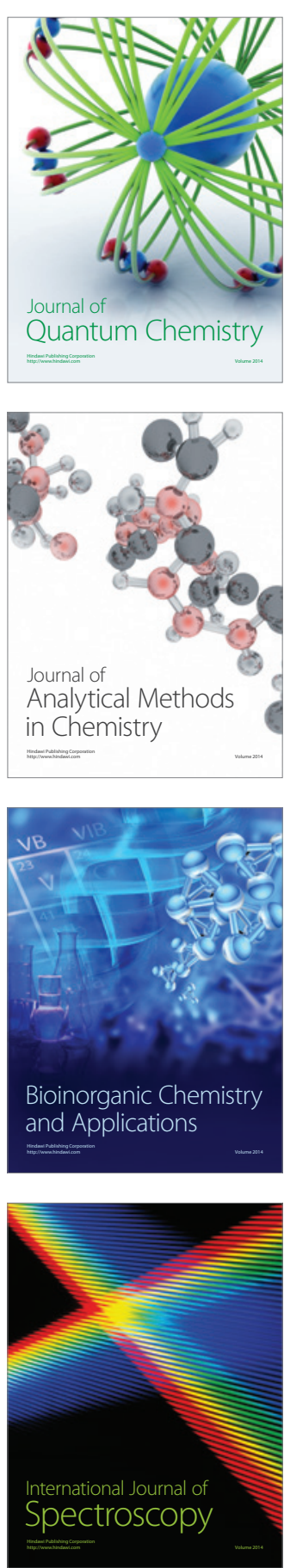